\title{
Free-electron response in reflectance anisotropy spectra
}

\author{
K. Fleischer, ${ }^{1,2, *}$ S. Chandola, ${ }^{1,2}$ Th. Herrmann, ${ }^{2}$ N. Esser, ${ }^{3}$ W. Richter,,${ }^{2,4}$ and J. F. McGilp ${ }^{1}$ \\ ${ }^{1}$ School of Physics, Trinity College Dublin, Dublin 2, Ireland \\ ${ }^{2}$ Technische Universität Berlin, Institut für Festkörperphysik, Sekr. PN 6-1, Hardenbergstrasse 36, D-10623 Berlin, Germany \\ ${ }^{3}$ ISAS-Institute for Analytical Sciences, Department Berlin, Albert-Einstein Strasse 9, 12489 Berlin, Germany \\ ${ }^{4}$ INFM, Universitá di Roma Tor Vergata, Via della Ricerca Scientifica 1, I-00133 Roma, Italy
}

(Received 28 March 2006; revised manuscript received 3 October 2006; published 27 November 2006)

Reflectance anisotropy spectra (RAS), extended into the near infrared spectral region, of anisotropic metal islands, metal surfaces, and atomic nanowires are analyzed with respect to anisotropies in the optical response of the free-electron gas of such low dimensional metal structures. In order to distinguish conductivity and morphology effects, both a phenomenological anisotropic Drude model and an effective medium model using ellipsoidal metal inclusions are used to model the spectra. In some cases (metal islands) the RAS response is dominated by the free-electron contribution, while this contribution is significantly smaller for the metal surfaces and nanowires in the accessible spectral range.

DOI: 10.1103/PhysRevB.74.195432

PACS number(s): 73.50. $-\mathrm{h}$, 73.20.At, 73.63.Nm, 73.25.+i

\section{INTRODUCTION}

In this work the contribution of the free-electron gas to the reflectance anisotropy spectra (RAS) of low dimensional metallic nanostructures or nanowires will be addressed. Interest in such metallic nanowires and their basic properties has increased in recent years, particularly for wires on surfaces with widths of a few atoms. These can show interesting effects such as Peierls instabilities and charge density waves $(\mathrm{CDW})$, and are also promising candidates for investigating more exotic effects such as spin charge separation. ${ }^{1-4}$ So far, investigations have been mainly performed using electron based methods such as scanning tunnelling microscopy and spectroscopy (STM, STS), and angle resolved photoemission spectroscopy (ARPES). Due to the limited penetration depth of electrons, these methods are highly surface sensitive but are only suited for nanostructures on surfaces, or the surfaces themselves. This is a problem for characterizing metallic nanowires in devices, since the properties of the wire need to be known even if they are buried below protective caps to ensure stability in ambient conditions. One possible way to overcome this limitation is to use surface or interface sensitive optical probes, as the larger penetration depth of light allows buried structures to be accessed.

One prominent optical technique with such surface and interface sensitivity is reflectance anisotropy spectroscopy (RAS). The technique itself was introduced in the mid $1980 \mathrm{~s}^{5-7}$ and is nowadays used in both surface science and in semiconductor technology [as growth control in gaseous environments, such as in metal organic vapor phase epitaxy (MOVPE), where electron based methods cannot be applied $^{8,9}$. The origin of the RAS response from anisotropic surfaces is quite well understood and can be calculated $a b$ initio within density functional based theories. ${ }^{10,11}$ The main structures in the RAS spectra arise from transitions between surface states or surface modified bulk states. RAS is well suited for the analysis of metallic nanowires and has proved to be a useful tool in monitoring nanowire formation ${ }^{12,13}$ or Peierls driven metal-insulator transitions in metallic nanowires. ${ }^{14}$ For such systems the spectral signature of the interband electronic transitions has already been discussed. ${ }^{15}$

In contrast to these earlier studies, this paper focuses on the intraband transitions and the direct observation of a conductance anisotropy with RAS. Experimentally the basic idea is to observe these contributions in the near infrared (IR) spectral region, similar as it has been done for the freecarrier contribution to the dielectric function (absorption), where the intraband contributions start to dominate. ${ }^{16,17}$ It will be shown that this response can be modeled by using a Drude-type dielectric function with two independent tensor components, $\widetilde{\varepsilon}_{x}$ and $\widetilde{\varepsilon}_{y}$, contributing to the anisotropic RAS signal. This simple model appears promising in relating the measured spectra of metallic nanowires to the conductance anisotropy, thus facilitating contactless measurements of this quantity. Theoretical $a b$ initio calculations of the interband anisotropy from the (110)-surface of $\mathrm{Cu}$ and $\mathrm{Al}$ (Ref. 18) already indicate that such contributions to the reflectance anisotropy exist.

\section{DETAILS OF THE OPTICAL MODELS}

RAS measures the difference in the reflectance of light polarized along two orthogonal axes $x$ and $y$ in the surface plane, normalized to the mean reflectance.

$$
\frac{\Delta R}{R}=2 \frac{R_{x}-R_{y}}{R_{x}+R_{y}} .
$$

If the complex Fresnel reflection coefficient, $\widetilde{r}$, can be measured, then

$$
\frac{\Delta \widetilde{r}}{\tilde{r}}=2 \frac{\widetilde{r}_{x}-\widetilde{r}_{y}}{\widetilde{r}_{x}+\widetilde{r}_{y}}
$$

As the anisotropy is normally small, $\Delta R / R \approx 2 \operatorname{Re}(\Delta \widetilde{r} / \widetilde{r})$. Both $\Delta R / R$ and $\operatorname{Re}(\Delta \widetilde{r} / \widetilde{r})$ have been termed RAS signals in the literature: ${ }^{7}$ here only the latter will be used.

\section{A. The surface dielectric anisotropy}

As no $a b$ initio calculations are available for the measured systems in the IR spectral region, the experimental results are 
compared with calculations within a three layer model, ${ }^{19}$ comprising an isotropic bulk with dielectric function $\widetilde{\varepsilon}_{b}$, a modeled effective anisotropic layer for the nanowires $\left(\widetilde{\varepsilon}_{x}, \widetilde{\varepsilon}_{y}\right)$, and finally air as the ambient layer. The RAS can then be expressed as

$$
\frac{\Delta \widetilde{r}}{\widetilde{r}}=\frac{4 \pi i d}{\lambda} \frac{\widetilde{\varepsilon}_{x}-\widetilde{\varepsilon}_{y}}{\widetilde{\varepsilon}_{b}-1},
$$

where $d$ is the effective thickness of the anisotropic layer. ${ }^{5}$ The quantity $d\left(\widetilde{\varepsilon}_{x}-\widetilde{\varepsilon}_{y}\right)$ is called the surface dielectric anisotropy (SDA) and is a robust quantity: in the absence of other information, the division between $d$ and $\left(\widetilde{\varepsilon}_{x}-\widetilde{\varepsilon}_{y}\right)$ is uncertain. With Eq. (3) it is possible to calculate the RAS spectra using model dielectric functions for comparison with experiment or, since $\widetilde{\varepsilon}_{b}$ is usually known, to discuss RAS spectra in terms of the dielectric anisotropy by calculating the SDA from the measured spectra.

For the measurements presented here, the three layer description is sufficient. In the case of buried structures more complex models have to be used: for example, the dielectric anisotropy of the surface and interface have been successfully separated for AlAs/GaAs heterostructures. ${ }^{20}$ In this initial study only uncapped wires are analyzed and the simple three layer model is sufficient.

Frequently RAS spectra show structure close to bulk critical points of the substrate which partly originate from the normalization by $\widetilde{\varepsilon}_{b}$ in Eq. (3) and partly by interface modified bulk states. To distinguish these from the response of the nanowires the bulk critical points are marked in all figures, e.g., $E_{1}, E_{1}+\Delta_{1}, E_{0}^{\prime}$, and $E_{2}$ in Fig. 3.

\section{B. Model dielectric functions}

In order to describe the dielectric function of the metallic nanowire two different approaches will be compared. The first utilizes a three-dimensional (3D)-Bruggemann effective medium approximation (EMA) assuming an ellipsoid shape of the wire. The anisotropic layer is then described by a modified, anisotropic Bruggeman EMA, using a mixture of the ellipsoids of bulk metal dielectric function and vacuum. ${ }^{21}$ The percolation threshold is $f=1 / 3$ for spherical inclusions in this model although, in its anisotropic formulation using ellipsoids, $f$ itself is anisotropic, with larger values in the direction of the long axis of the ellipsoid and smaller values along the short axis. ${ }^{21}$ This approach has been used successfully for modeling the effect of roughness on RAS spectra of clean semiconductor surfaces. ${ }^{22}$ As will be seen later, this approach works well for larger anisotropic islands, but fails to describe the case for wires with diameters smaller than $50 \mathrm{~nm}$. Instead a simpler, phenomenological approach can be used, where only anisotropy in the free-electron properties are assumed.

The dielectric function of a free-electron gas can be described using the Drude-Lorentz formulas for an oscillator with eigenfrequency $\omega_{0}=0$ :

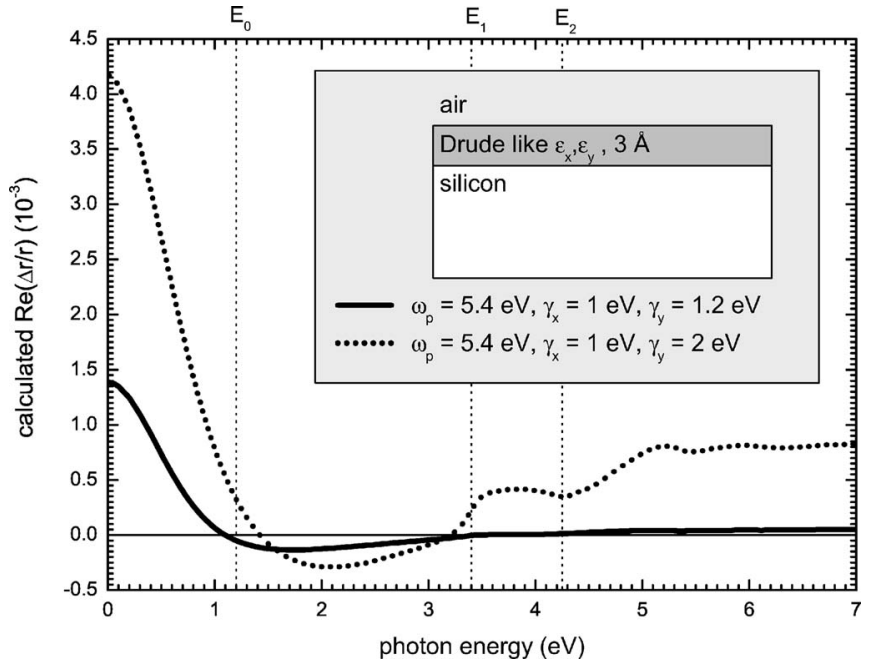

FIG. 1. Model RAS spectra of an anisotropic Drude layer on silicon. The solid line shows a calculation with an anisotropy $\gamma_{y} / \gamma_{x}=1.2$, the dashed line one with $\gamma_{y} / \gamma_{x}=2$. The anisotropic freeelectron response is mainly seen in the IR, while the bulk critical points of the substrate can be observed in the UV region. The anisotropic $\gamma$ values lead to a sign change in the RAS response. Energy units are used for all the parameters.

$$
\varepsilon^{\prime}=1-\frac{\omega_{p}^{2}}{\omega^{2}+\gamma^{2}}, \quad \varepsilon^{\prime \prime}=\frac{\omega_{p}^{2} \gamma}{\omega\left(\omega^{2}+\gamma^{2}\right)}
$$

where $\widetilde{\varepsilon}=\varepsilon^{\prime}-i \varepsilon^{\prime \prime}$. In these formulas $\gamma(=1 / \tau)$ is the scattering rate which is related to damping with a phenomenological scattering time of $\tau$. In the Drude theory, $\omega_{p}$ is given by $\omega_{p}^{2}=N_{c} e^{2} /\left(m_{e} \varepsilon_{0}\right)$ where $N_{c}$ is the free-electron density and $m_{e}$ the free-electron mass. Although originally derived for noninteracting electrons in $3 \mathrm{D}$, the Drude dielectric function nevertheless agrees well with the measured dielectric function of bulk metals. ${ }^{23-26}$

The optical anisotropy in a free-electron gas may thus be caused by anisotropic values of the effective masses (replacing the free-electron mass) and/or scattering rates. As a first approximation, the experimental bulk plasma frequency is used (implying an isotropic effective mass), while different values for the scattering rate are assumed here for the two orthogonal directions probed with the RAS. This seems reasonable, as scattering in metals at room temperature is a nanoscale phenomenon, allowing surface and interface scattering from anisotropic nanoscale structures to produce anisotropic scattering rates. This will be discussed further in Sec. VII. The set of parameters $\left(\omega_{p}, \gamma_{x}, \gamma_{y}\right)$ appears sufficient to describe the infrared SDA. For RAS model calculations, the thickness of the layer $d$ is needed as an additional parameter. Figure 1 shows an example of a model RAS calculation of such an anisotropic Drude layer on silicon, where the effect of varying the anisotropic scattering rate is shown.

Such a contribution to the RAS of metallic structures has been discussed but, due to the limited spectral response of most RAS systems, the contribution was always found to be too weak for quantitative analysis. ${ }^{27-29}$ As will be shown here, metallic nanowires on semiconductor surfaces can pro- 
duce a stronger response, particularly in the energy region below $1 \mathrm{eV}$, which is accessible with the IR enhanced RAS spectrometer described below.

\section{Anisotropic conductivity}

As the IR reflectance anisotropy of a 1D metallic system can be related to a free-electron contribution, the question arises whether conclusions about the conductivity can be drawn from RAS spectra as well. The dielectric function is related to the optical conductivity, $\widetilde{\sigma}:{ }^{19,30}$

$$
\widetilde{\varepsilon}=1-i \widetilde{\sigma} / \varepsilon_{0} \omega .
$$

The RAS signal can be described in terms of a conductivity anisotropy, $\Delta \widetilde{\sigma}=\widetilde{\sigma}_{x}-\widetilde{\sigma}_{y}$, by using this relation and the three layer model of the RAS signal from Eq. (3):

$$
\frac{\Delta \widetilde{r}}{\tilde{r}}=\frac{2 d}{\varepsilon_{0} c} \frac{\Delta \widetilde{\sigma}}{\widetilde{\varepsilon}_{b}-1} .
$$

Hence the RAS signal is directly proportional to this conductivity difference. The RAS spectra allows $d \Delta \widetilde{\sigma}$ to be determined, analogously to the SDA. This is particularly useful, as $d \Delta \widetilde{\sigma}$ is the anisotropy in the sheet conductance determined by conventional four-point electrical measurements, assuming the underlying bulk does not contribute significantly. Fitting the infrared RAS using the anisotropic Drude model allows $\Delta \widetilde{\sigma}$ to be determined at zero frequency, thus giving an estimate of the dc conductivity. Such a calculation corresponds to an extrapolation of a measured ac conductivity to $\omega=0$. Infrared transmittance spectroscopy has been used previously to determine the conductivity of $2 \mathrm{D}$ films: ${ }^{31,32}$ it is shown here that IR enhanced RAS is sensitive to the conductivity anisotropy of $1 \mathrm{D}$ structures.

\section{EXPERIMENT}

The RAS instruments follow the basic design of a two polarizer system and photoelastic modulator (PEM), as proposed by Aspnes et al. ${ }^{7}$ One broad range RAS system $(0.75-6.5 \mathrm{eV})$ and one IR optimized system $(0.5-4.5 \mathrm{eV})$ were used for the measurements presented here. Both systems utilize multiple detectors: InGaAs/Si diodes and photomultiplier in the first case, and a combination of liquid nitrogen cooled InAs photodiode and $\mathrm{InGaAs}_{\mathrm{SS}}$ diodes in the latter case. In order to access such a broad spectral range, both systems were equipped with either two independent monochromators or a double grating monochromator. All optical components were chosen for broad range performance with, for example, $\mathrm{MgF}_{2}$ polarizers and $\mathrm{CaF}_{2}$ PEMs being used. A Xe arc discharge lamp was normally used, although for some IR measurements this was replaced by a quartz halogen lamp, although this limits the range to $0.5-3.0 \mathrm{eV}$.

The sample preparation was always performed under ultrahigh vacuum conditions at a base pressure below $1 \times 10^{-10}$ mbar. The InAs(110) surfaces were prepared by in situ cleavage, the $\mathrm{Si}(111)$ by removing the native oxide layer at $1100{ }^{\circ} \mathrm{C}$. The metal surfaces were cleaned by sputter and annealing cycles. For the preparation of the Sn and In nanowires, the metals were deposited onto the clean surfaces from (a)

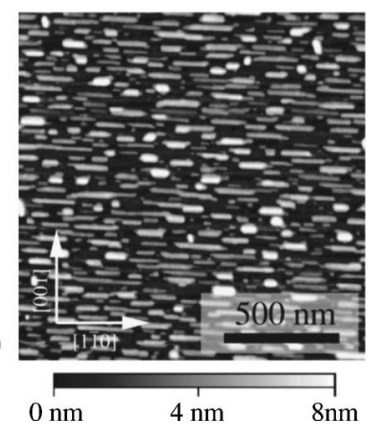

(b)

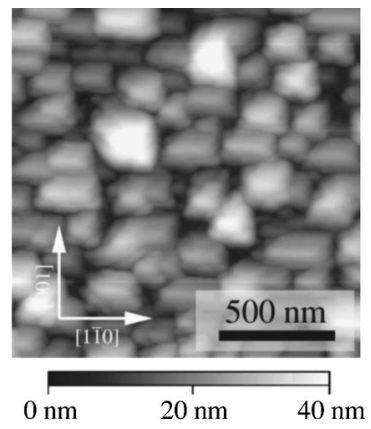

FIG. 2. AFM images of (a) $20 \mathrm{ML}$ and (b) $100 \mathrm{ML}$ of Sn on InAs(110). For smaller coverages the tin islands are highly anisotropic with a long axis of up to $400 \mathrm{~nm}$ and a short axis of only $25 \mathrm{~nm}$. At higher coverages the islands are larger but more isotropic (400 nm long, $100 \mathrm{~nm}$ wide).

Knudsen-type evaporator cells. In the case of $\mathrm{Sn} / \operatorname{InAs}(110)$, the deposition was performed at room temperature and was controlled with a quartz microbalance, calibrated using Auger electron spectroscopy (AES) and ex situ atomic force microscopy (AFM) measurements of the average island height. In the case of the $\mathrm{In} / \mathrm{Si}(111)$ system the sample was kept at $450{ }^{\circ} \mathrm{C}$ during the indium deposition and the formation of the indium nanowires was monitored directly by the RAS measurement. The presence of the single domain $(4 \times 1)$ reconstruction of surface was checked afterwards with low energy electron diffraction (LEED).

\section{ANISOTROPIC METAL ISLANDS}

Two systems of metallic nanostructures are discussed here. Anisotropic islands of $\beta$-tin grow on $\operatorname{InAs}(110)$, and produce large infrared RAS structures. The second system, which also grows anisotropic metal islands, is $\mathrm{Pb} / \mathrm{Si}(335)$, which was investigated by Stròżak et al. with another optical technique, surface difference spectroscopy (SDR). ${ }^{33}$ This interesting work includes sufficient polarization dependent measurements to enable a RAS response to be calculated from the data, for comparison with the anisotropic Drude model.

\section{A. $\operatorname{Sn} / \operatorname{InAs}(110)$}

Anisotropic islands of $\beta$-tin were grown on cleaved InAs(110) surfaces at room temperature. In Fig. 2 atomic force micrographs, taken ex situ, are shown for nominal thicknesses of 20 and $100 \mathrm{ML}$ of Sn. The islands become more isotropic as the coverage increases. By $100 \mathrm{ML}$, large rectangular islands of $\beta$-tin have been formed. Although the exact crystallographic orientation is not known, the shape anisotropy of the islands suggest that the tetragonal $c$-axis is within the surface plane.

In Fig. 3 the RAS spectra of a 40 and a 60 ML sample are shown. The spectra are dominated by the bulk Sn response, although there is still some structure in the region of the bulk critical points of the InAs substrate (labeled), which are major features in the RAS spectra at very low coverages. The spectra can be qualitatively understood by an anisotropic 3D- 


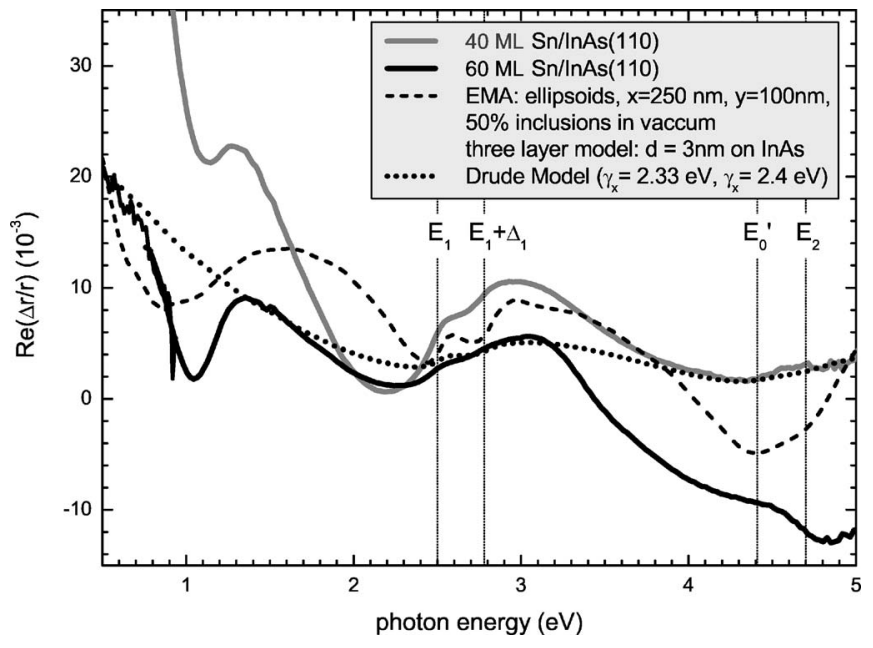

FIG. 3. RAS spectra of $40 \mathrm{ML}$ (gray line) and $60 \mathrm{ML}$ (black line) coverage of Sn. The general line shape of the $60 \mathrm{ML}$ sample can be modeled using the bulk properties of Sn and an anisotropic effective media approximation (dashed line). The strong infrared RAS signal in the $40 \mathrm{ML}$ spectrum (and at lower coverages) cannot be explained with such effective medium models. For comparison a least-squares fit using the anisotropic Drude model is also shown. The regions with strong $\mathrm{Sn}$ interband contributions were excluded in the fit.

Bruggeman effective medium approximation (EMA) with oriented ellipsoidal inclusions ${ }^{21,22}$ of bulk $\mathrm{Sn}$, as shown by the dashed curve in Fig. $3(d=3 \mathrm{~nm}, x=250 \mathrm{~nm}$, $y=100 \mathrm{~nm}, f=0.5$, where $f$ is the filling fraction). The effective dielectric function is individually calculated for light polarized along, and perpendicular to, the long axis $\left(\widetilde{\varepsilon}_{x}, \widetilde{\varepsilon}_{y}\right)$. The AFM images were used to determine values for $x$ and $y$, while the thickness, $d$, in Eq. (3) was fitted to obtain best agreement with the measurement. It is clear that this approximation fails to describe the strong IR increase occurring at lower coverage, but provides qualitative agreement with the general line shape of the $60 \mathrm{ML}$ spectra. The agreement is reasonable, considering that the bulk dielectric function used in these calculation was derived from microcrystalline samples with random orientation. ${ }^{34}$ The islands here are clearly oriented, and the much sharper structure around $1 \mathrm{eV}$ in the measured RAS response probably arises from an anisotropic absorption in the tin, which is broadened in the measured $\widetilde{\varepsilon}_{b, \text { Sn }}$ of the randomly oriented crystals. Additionally, a strong broad minimum in the $3-5 \mathrm{eV}$ region evolves for higher coverages which is part of a Mie resonance occurring for large islands. Such structures were already seen and successfully simulated for larger indium islands on GaAs. ${ }^{35}$ In the case of the $60 \mathrm{ML}$ sample a fit using the anisotropic Drude model does not provide any new information. Although it does agree well in the IR when the areas of strong interband transition are excluded, the simple morphological model using the bulk Sn dielectric function and an anisotropic EMA provides equally good agreement.

For smaller coverages $(<40 \mathrm{ML})$, where the anisotropies in the island geometry are much more pronounced, the EMA model is not sufficient to reproduce the RAS line shape (see inset Fig. 4). In particular, the strong signal in the IR

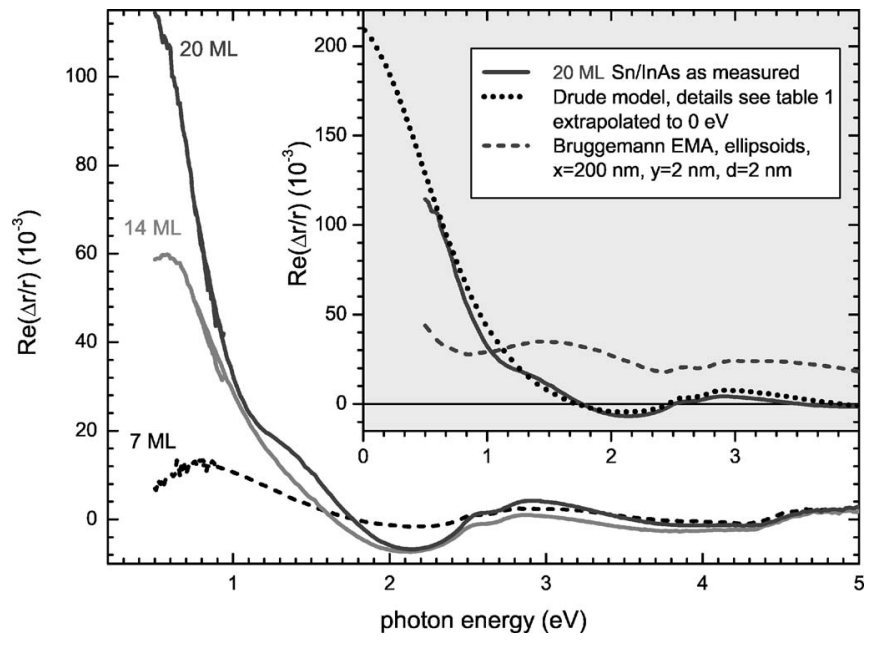

FIG. 4. In the coverage region between 5 and $40 \mathrm{ML}$, the reflectance anisotropy of tin islands on $\operatorname{InAs}(110)$ is dominated by a strong IR structure. The strongest anisotropy was found for a coverage of $25 \mathrm{ML}$. The inset compares the anisotropic EMA model, using the Sn bulk $\widetilde{\varepsilon}$ (dashed line) and Drude model (dotted line). In contrast to thicker layers, the EMA model fails to describe the measurements, while the Drude model agrees well with the measured data.

regime cannot be explained, while the simpler Drude model reproduces the results very well.

The IR structure emerges for coverages above 1.5 ML and shows a peak at $1.3 \mathrm{eV}$ that redshifts with increasing coverage $(0.8 \mathrm{eV}$ at $7 \mathrm{ML} ; 0.6 \mathrm{eV}$ at $14 \mathrm{ML})$. This behavior could arise from a number of factors. First, changes in gradient in the IR tail of the two orthogonal polarizations may produce a peak when the difference is plotted. Second, if the metallic layer is above the percolation threshold, the Drude-like dielectric response may not increase monotonically to lower energies. ${ }^{32,36}$ Third, EMA models using elliptical inclusions produce a percolation threshold that depends on the ellipticity, which changes significantly with coverage (see Fig. 2). Detailed in situ studies of the morphology in this coverage regime would be necessary to clarify the importance of these various contributions.

For higher coverages $(>14 \mathrm{ML})$ the IR response at $0.8 \mathrm{eV}$ is maximal at $25 \mathrm{ML}$ and then decreases until the morphological anisotropy dominates the spectra above $40 \mathrm{ML}$, as discussed above. The quality of agreement achieved with the anisotropic Drude model is shown for a $20 \mathrm{ML}$ sample in the inset of Fig. 4. Least-square fits of model parameters in this coverage region give sufficiently good agreement that quantitative information can be extracted from the RAS spectra. The number of free parameters is minimized by using known values where possible. The thickness, $d$, was set to the nominal layer thickness, and $\omega_{p}$ was set to the $\beta$-tin bulk value of $13 \mathrm{eV} .{ }^{34}$ The remaining two parameters $\left(\gamma_{x}, \gamma_{y}\right)$ were determined by least-squares fits of the SDA (see Fig. 5). The best agreement is obtained in the range 10-30 ML. In Table I the results are summarized and an estimation of the anisotropy in the dc conductivity is given by extrapolating the Drude-like RAS response to zero frequency. It is important to note that these predictions can only account for the free-electron con- 


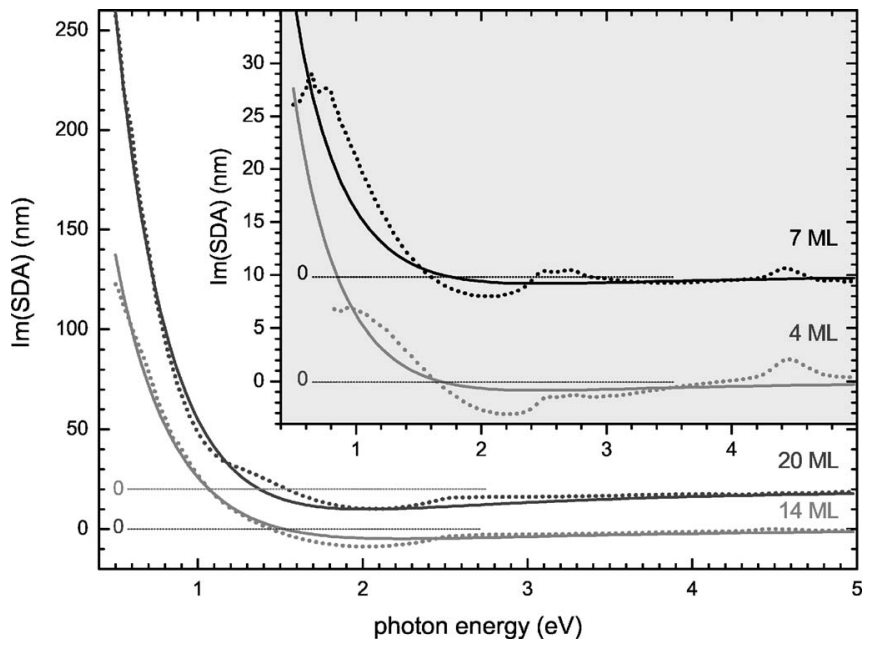

FIG. 5. Experimental surface dielectric anisotropies for various coverages, determined from the RAS measurements (dotted curves). The spectra are stacked for better visibility. The solid lines show the best fits to the anisotropic Drude model. The values from these fits are given in Table I.

tribution to the conductance and also neglect any percolation effects.

Table I shows that, as expected, the scattering rate is uniformly higher in the shorter, $y$ direction of the anisotropic $\mathrm{Sn}$ islands. Also, the values of the anisotropy in the sheet conductance appear reasonable. The sheet conductance, estimated using the bulk conductivity of tin and the layer thickness for the $20 \mathrm{ML}$ sample, gives $32 \times 10^{-3} \mathrm{~S} / \square$, while the measured anisotropy in the conductance is $2.3 \times 10^{-3} \pm 0.5$ $\times 10^{-3} \mathrm{~S} / \square$. The fractional anisotropy of 0.04 is a lower limit because the conductivity of such a thin granular film is expected to be significantly lower than the bulk value. ${ }^{37}$ For example, if the measured values for Sn layers $(>5 \mathrm{~nm})$ on glass $^{38}$ are extrapolated to the thickness of the $20 \mathrm{ML}$ sample, a value of $d \sigma$ of $6 \times 10^{-3} \mathrm{~S} / \square$ is obtained, giving a fractional anisotropy of 0.38 . The values for $d \Delta \sigma$ in Table I, which vary from $0.2 \times 10^{-3}$ to $2.3 \times 10^{-3} \mathrm{~S} / \square$, are clearly reasonable.
It should be noted that the fitted value obtained for the anisotropic sheet conductance is relatively robust to parameter changes. For example, if $\omega_{p}$ is set to the surface plasmon frequency $\omega_{p, s}=\omega_{p, b} / \sqrt{2}$, equally good fits can be derived, which have smaller scattering rates but larger anisotropies: for the $20 \mathrm{ML}$ sample, $\gamma_{x}=0.93 \mathrm{eV}$ and $\gamma_{y}=2.1 \mathrm{eV}$ is found, but the actual line shape of the alternative fits, and therefore also the dc sheet conductance anisotropy, remains the same.

\section{B. $\mathrm{Pb} / \mathrm{Si}(335)$}

The $\mathrm{Pb} / \mathrm{Si}(335)$ system allows the growth of isolated and aligned $\mathrm{Pb}$ nanoscale islands, which are sufficiently anisotropic to be described as nanowires. Although no RAS measurements currently exist for this particular system, the surface differential reflection (SDR) is known for polarization along, $\mathrm{SDR}_{x}$, and perpendicular to, $\mathrm{SDR}_{y}$, the wire axis. ${ }^{33}$ The RAS spectrum can be determined from the published SDR data, assuming that the reflectivity of the clean surface is isotropic, $R_{\mathrm{Si}, x}=R_{\mathrm{Si}, y}$ (this is a reasonable assumption in the IR regime, well below the $\mathrm{Si} E_{1}$ bulk critical point).

$$
\begin{gathered}
\mathrm{SDR}_{x}=\frac{R_{\mathrm{Si}+\mathrm{Pb}, x}-R_{\mathrm{Si}, x}}{R_{\mathrm{Si}, x}}, \\
\mathrm{SDR}_{y}=\frac{R_{\mathrm{Si}+\mathrm{Pb}, y}-R_{\mathrm{Si}, y}}{R_{\mathrm{Si}, y}}, \\
\left.\frac{\Delta R}{R}\right|_{R A S}=2 \frac{R_{\mathrm{Si}+\mathrm{Pb}, x}-R_{\mathrm{Si}+\mathrm{Pb}, y}}{R_{\mathrm{Si}+\mathrm{Pb}, x}+R_{\mathrm{Si}+\mathrm{Pb}, y}} \\
=2 \frac{\mathrm{SDR}_{x}-\mathrm{SDR}}{\mathrm{SDR}_{x}+\mathrm{SDR}_{y}} .
\end{gathered}
$$

In Fig. 6, RAS spectra and SDA, determined from the SDR data of Stròżak et al. ${ }^{33}$ using Eq. (9), are shown for a coverage of $6 \mathrm{ML}$. A strong RAS signal in the IR is observed, accompanied by a sign reversal in the near IR. This is an encouraging sign that, again, the main contribution to the

TABLE I. Free-electron parameters derived by fitting the SDA for various coverages of Sn on InAs(110). Parameters which were kept fixed are marked. The confidence interval of the fitted values were below $0.05 \mathrm{eV}$ if not explicitly stated. For $40 \mathrm{ML}$ the region from 1 to $1.5 \mathrm{eV}$ was excluded from the fit because of the strong influence of interband transition in this regime. The last values given are the RAS signal linearly extrapolated to $\omega=0\left(\Delta r /\left.r\right|_{\mathrm{dc}, \text { meas }}\right)$ and the calculated value for $\omega=0$ from the given Drude parameters $\left(\Delta r /\left.r\right|_{\mathrm{dc} \text {,calc }}\right)$. The anisotropic dc sheet conductance was calculated with the fitted Drude parameters using Eq. (6) and is given here as $d \Delta \sigma$.

\begin{tabular}{lcccccc}
\hline \hline Thickness & in ML & 4 & 7 & 14 & 20 & 40 \\
\hline$d$ (fixed) & in nm & 0.7 & 1.3 & 2.5 & 3.5 & 7.2 \\
$\omega_{p}$ (fixed) & in eV & 13 & 13 & 13 & 13 & 13 \\
$\gamma_{x}$ & in eV & $1.5 \pm 0.08$ & $2.1 \pm 0.1$ & 1.30 & 1.13 & 1.77 \\
$\gamma_{y}$ & in eV & $1.9 \pm 0.08$ & $2.4 \pm 0.1$ & 1.78 & 1.67 & 2.02 \\
$\gamma_{y} / \gamma_{x}$ & & 1.26 & 1.14 & 1.38 & 1.48 & 1.14 \\
$\Delta r /\left.r\right|_{\text {dc,calc }}$ & & 0.02 & 0.02 & 0.11 & 0.21 & 0.06 \\
$\Delta r /\left.r\right|_{\text {dc,meas }}$ & & 0 & 0 & 0.05 & 0.13 & 0.08 \\
$\operatorname{Re}\left(\left.d \cdot \Delta \sigma\right|_{\text {dc }}\right)$ & in $10^{-3} \mathrm{~S}$ & $0.2 \pm 0.05$ & $0.2 \pm 0.05$ & $1.2 \pm 0.2$ & $2.3 \pm 0.5$ & $1.2 \pm 0.4$ \\
\hline \hline
\end{tabular}




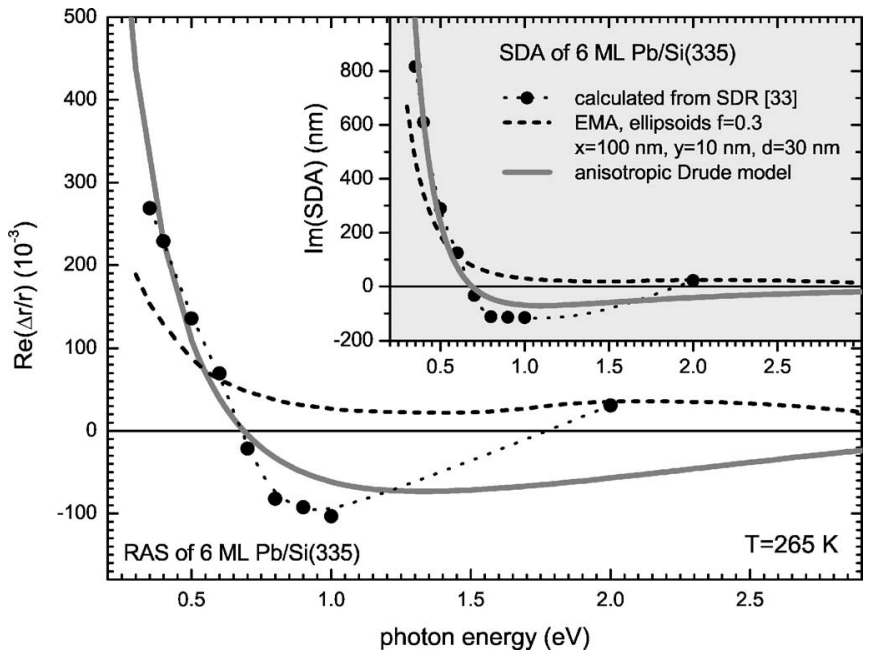

FIG. 6. RAS spectra of $6 \mathrm{ML} \mathrm{Pb}$ on $\mathrm{Si}(335)$, determined from the SDR values of Stròżak et al. using Eq. (9). The inset shows the corresponding surface dielectric anisotropy. Although only few data points exist, the strong IR signal and a sign change of the RAS can be seen. The former can, in principle, also be explained by an effective medium model (Bruggeman with ellipsoid inclusions, dashed line), but not the latter. The anisotropic free-electron model (gray line: $\omega_{p}=7.8 \mathrm{eV}$ and $\gamma_{x}=0.2 \mathrm{eV}$ [bulk values (Ref. 26)] and fitted $\gamma_{y}=2.36 \pm 0.4 \mathrm{eV}$ and $d=6.8 \pm 0.7 \mathrm{~nm}$ ) can model both features.

signal arises from an anisotropic free-electron response and, in particular, an anisotropic scattering rate.

A low surface density of isolated islands is produced at these coverages, with a height much larger than the nominal thickness, in contrast to the simpler case of the tin islands. For example, the island height is explicitly stated to be $13 \mathrm{~nm}$ under the particular preparation conditions for a $3 \mathrm{ML}$ sample. ${ }^{33}$ Thus $d$ was kept as a free parameter, together with $\gamma_{y}$, while $\omega_{p}$ and $\gamma_{x}$ were fixed at the bulk values. ${ }^{26}$ A thickness of $6.8 \mathrm{~nm}$ was obtained for the $6 \mathrm{ML}$ sample, which is about five times larger than the nominal thickness, but is significantly smaller than the expected island height based on the $3 \mathrm{ML}$ data. The thickness, $d$, refers to the effective optical layer used to describe the highly percolated system of a few high $\mathrm{Pb}$ islands sitting on a metallic wetting layer and hence cannot be directly interpreted as either the average height of the islands or the average thickness of the $\mathrm{Pb}$, in contrast to the much more dense morphology of the Sn islands.

As the spectral region is relatively narrow and only a few data points are available, the parameter values of the anisotropic Drude model are less certain than in the case of the Sn islands discussed above: for example, the anisotropic scattering ratio of 10 seems rather high. However, Fig. 6 shows that the overall line shape can be reasonably reproduced with the anisotropic Drude model. In contrast, the EMA model, using the $\mathrm{Pb}$ bulk $\widetilde{\varepsilon}$, cannot account for the strong IR signal. By using a larger plasma frequency, the Drude model agreement below $1 \mathrm{eV}$ can be improved, though the discrepancy at the $2 \mathrm{eV}$ data point increases. The experimental value of the anisotropy at $2 \mathrm{eV}$, derived from the SDR measurements, does not vary much with coverage, which is surprising, and $\mathrm{Pb}$ interband transitions occur in this spectral range, leading to the conclusion that the $2 \mathrm{eV}$ point is unlikely to be determined predominantly by the free electron response. It remains possible that the behavior in the high energy region could arise from percolation effects. ${ }^{36,39}$ The morphology of the wires is better known for this system than for $\mathrm{Sn} / \mathrm{InAs}$, but there are insufficient data points to explore this possibility.

Summarizing, a strong infrared RAS signal occurs in two anisotropic metal island systems grown on semiconducting surfaces, with a higher reflectance for light polarized along the long axis of the islands. The signal occurs for both densely packed islands and for isolated wires. The line shape of this signature can be reproduced quite well by a three layer model, where the surface layer is modeled by two independent Drude-like dielectric functions, which allows an anisotropic scattering rate to be introduced, with the lower scattering rate being associated with the long axis of the islands. It thus appears possible to probe anisotropic free electron properties at such surfaces optically, including estimating a value of the anisotropy in the dc sheet conductance in favorable cases.

\section{ATOMIC NANOWIRES}

Quasi-one-dimensional systems, which are much smaller than the island structures discussed so far, can be prepared on surfaces by self-organized growth. Such nanowires may be only four atoms wide and still show metallic properties, as in the case of indium wires on $\mathrm{Si}(111)$ - the $\mathrm{Si}(111$ : In-(4 $\times 1)$ ) reconstruction. There are numerous other systems where a metal adsorbate forms one-dimensional structures on a surface, though not all of them show metallic properties: a superficially similar system, $\operatorname{Si}(111): \operatorname{Ag}-(3 \times 1)$, has a semiconducting surface. ${ }^{40,41}$

RAS measurements of the $\mathrm{Si}(111):$ In- $(4 \times 1)$ date back to 1998 and can only be done on the vicinal Si(111) surfaces that force single domain formation. ${ }^{13}$ The RAS spectrum (Fig. 7) is dominated by a strong interband transition at $2 \mathrm{eV}$. Nevertheless, the measurements show a small but significant IR anisotropy as well. Possible anisotropic freeelectron line shapes arising from the Drude model are shown in Fig. 8. In both graphs the thickness of the layer was assumed to be $d=1.5 \AA$, as the metallic surface state is reported to be located at the In-Si backbonds; ${ }^{42} \omega_{p}$ was approximated by the indium surface plasmon frequency. The remaining parameters, $\gamma_{x}=1.1 \pm 0.2 \mathrm{eV}$ and $\gamma_{y}=2.8 \pm 0.4 \mathrm{eV}$, were determined by fitting to the RAS data in the spectral region below $1.2 \mathrm{eV}$ and above $4.5 \mathrm{eV}$, away from the strong interband transitions.

The fitted $\gamma_{x}$ scattering rate compares well with the value given from a combined ARPES and conductivity study of $0.8 \mathrm{eV} .{ }^{43}$ In the same study the sheet conductance of $\mathrm{Si}(111):$ In- $(4 \times 1)$ has been measured along, and perpendicular to, the chain direction, giving $\Delta \sigma=7.1 \pm 0.6$ $\times 10^{-4} \mathrm{~S} / \square .{ }^{43}$ Assuming that the effective thickness of the dc conducting and Drude-type layers are equal, it is then possible, using Eq. (6), ${ }^{44}$ to calculate the RAS signal at $\omega=0$ as $50 \pm 4 \times 10^{-3}$. This is ten times larger than the extrapolated value. 


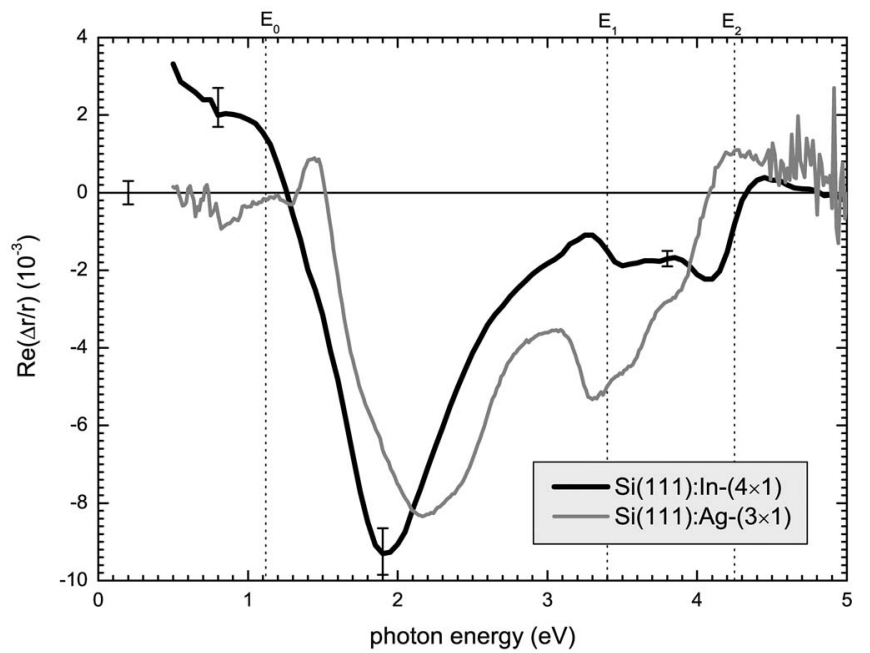

FIG. 7. RAS of the $\mathrm{Si}(111): \operatorname{In}-(4 \times 1)$ and $\mathrm{Si}(111): \mathrm{Ag}-(3 \times 1)$ surfaces (black and gray, line respectively). The spectra are dominated by anisotropic interband transitions around $2 \mathrm{eV}$. The experimental uncertainty of the zero line is indicated by the error bar. The sample-dependent variation of the $\operatorname{Si}(111): \operatorname{In}-(4 \times 1)$ RAS response (all showing a single domain $4 \times 1$ LEED pattern), is shown at 0.8 , 1.9 , and $3.8 \mathrm{eV}$.

If we include this known dc value as a datum in the fit (dashed line in Fig. 8), the much larger dc anisotropy can be accommodated, and the scattering rates become $\gamma_{x}=0.2 \pm 0.04 \mathrm{eV}$ and $\gamma_{y}=5.3 \pm 0.8 \mathrm{eV}$. The shaded area emphasizes the large change in the shape of the free-electron contribution between these two solutions and shows that, for this system, the dc conductivity cannot be determined reliably from RAS measurements above $0.5 \mathrm{eV}$.

The difficulty in extrapolating, successfully, the measured RAS response in this spectral range to zero frequency arises

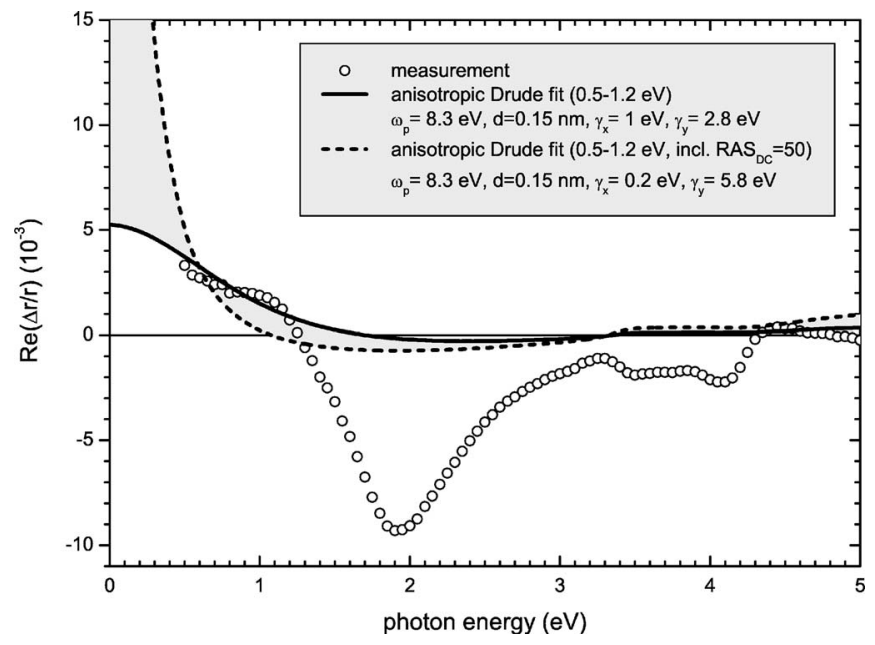

FIG. 8. Comparison of the $\operatorname{Si}(111): \operatorname{In}-(4 \times 1)$ RAS signal with the free-electron only response. All model parameters except $\gamma_{x}$ and $\gamma_{y}$ were kept fixed (see text) and only the spectral region below $1.2 \mathrm{eV}$ and above $4.5 \mathrm{eV}$ was included in the fit. For the solid line, only the RAS data were used in the fit, while, for the dashed line, the dc response determined from the measured anisotropy of the sheet conductance (Ref. 43) was included as a zero frequency datum.

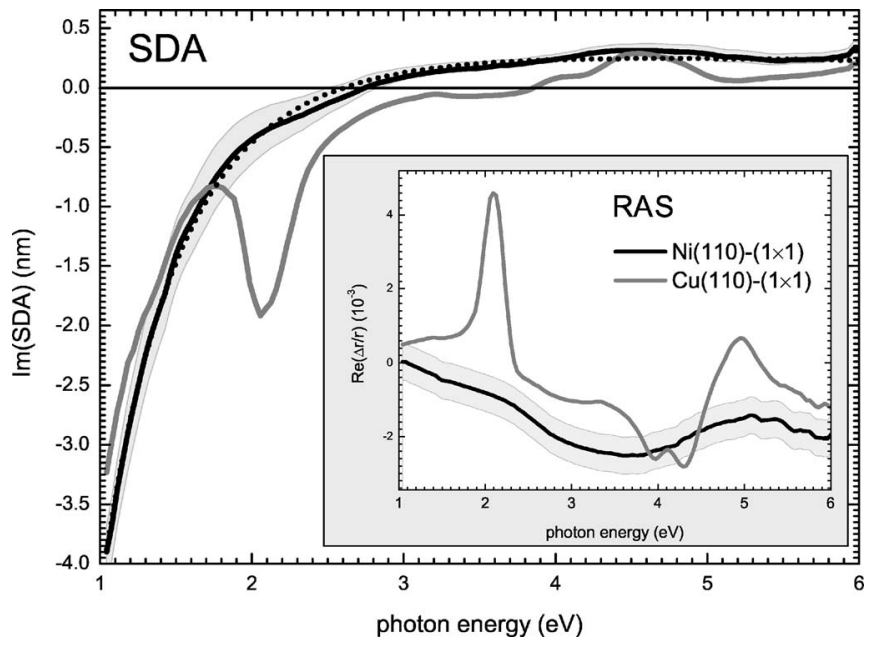

FIG. 9. Surface dielectric anisotropy of clean $\mathrm{Cu}(110)$ and $\mathrm{Ni}(110)$ surfaces. The inset shows the measured RAS spectra for the same surfaces. The SDA shows a strong resemblance to the expected Drude-like dielectric anisotropy for the $\mathrm{Ni}(110)$ surface (measurement—solid line, calculation-dotted line). The $\mathrm{Cu}(110)$ surface shows a similar Drude-like SDA with an additional peak at $2 \mathrm{eV}$ from the anisotropic surface state transition. For Ni(110) the error bar of the RAS zero line is \pm 0.5 , and the effect of this potential systematic error on the calculated SDA is indicated by the gray area.

from the combination of a small infrared RAS response and strong interband transitions. The small signal cannot be fitted over a sufficiently wide spectral range to produce reliable results, in contrast to the anisotropic island systems discussed above. There are indications that, for Si(111): In-(4 $\times 1$ ), interband transitions continue to contribute even below $1 \mathrm{eV}$. Samples with a maximized RAS signal at $1.9 \mathrm{eV}$ usually show a smaller anisotropy at $1 \mathrm{eV}$ (see bars in Fig. 7), and the response in this region also changes significantly in the presence of a small excess of In. ${ }^{14,45}$ It is clear from Fig. 8 , however, that a small extension of the spectral range to $0.3 \mathrm{eV}$ should allow a much more accurate estimate of the dc sheet conductance anisotropy.

In summary, the amplitude of the infrared RAS response is small for these atomic nanowires, but Fig. 7 shows that metallic and semiconducting structures can be distinguished optically. However, where strong interband transitions are present, it is clear that the spectral range has to be extended further into the IR if the anisotropy in the dc sheet conductance is to be determined, reliably, from the RAS response.

\section{CLEAN METAL SURFACES}

Anisotropic surfaces of clean metals should produce anisotropic electronic properties in the near surface region. $\mathrm{Cu}(110)$ and $\mathrm{Ni}(110)$ are two examples of anisotropic surfaces that have been studied by RAS. ${ }^{27,46,47}$ The RAS spectra of the $(1 \times 1)$ surfaces are shown in the inset of Fig. 9. The RAS spectra of the Ni surface is rather flat, while the $\mathrm{Cu}$ spectrum shows a sharp structure at $2 \mathrm{eV}$, due to a surface state and the onset of $d$-band transitions. In both cases the 
infrared RAS response is quite small. The influence of an anisotropic electron gas has been discussed in the case of the $\mathrm{Cu}(110)$ surface $^{27}$ and also for a similar metal surface, $\mathrm{Au}(110),{ }^{28}$ but in these studies the IR spectral range was not accessible and a free-electron contribution above $1.5 \mathrm{eV}$ was found to be negligible. Figure 9 shows that, in the RAS response (inset), no prominent structure arises in the IR, although the SDA line shapes, determined from the RAS spectra, show the characteristic Drude-like dielectric response. The SDA line shapes can be easily reproduced with the anisotropic Drude model (see dotted line in Fig. 9), apart from the interband transitions around 2 and $4.5 \mathrm{eV}$ for $\mathrm{Cu}(110)$, but there is a significant error associated with fitting these small anisotropies. An erroneous constant RAS signal, $C$ (zero line offset), which can easily occur in a two polarizer configuration, leads to a SDA of $C\left(\widetilde{\varepsilon}_{b}-1\right) \lambda / 4 \pi i$, from Eq. (3). Such zero line offsets are typically smaller than \pm 0.5 . The influence of such a systematic error is shown by the shaded area in Fig. 9.

Anisotropic roughness, usually modeled by effective media approaches, can also contribute to the dielectric anisotropy. ${ }^{48}$ For anisotropic islands on semiconducting surfaces, it has been shown above that EMA models, using the bulk metal $\widetilde{\varepsilon}$, fail to describe the strong IR structures and the characteristic zero line crossing of the SDA. In the case of metal surfaces, the SDA is smaller and the IR structure is comparable to the metal bulk critical point structures. Hence EMA models can also reproduce the measured SDA. However, for $\mathrm{Ni}(110)$, the small zero line crossing of the SDA indicates an anisotropic scattering rate contribution to the measured SDA, but morphological anisotropy cannot be completely excluded because the surface roughness, although likely to be very small on this single crystal surface, was not quantified.

Summarizing, the measured RAS signal in the accessible IR range from anisotropic metal surfaces is not dominated by a free-electron contribution because normalization by the bulk dielectric function of the metal $\widetilde{\varepsilon}_{b}$ in Eq. (3) drastically reduces the measured Drude-like response. The SDA calculated with Eq. (3) nevertheless does show a Drude-like line shape, which may arise from the near-surface anisotropic electron gas, but other contributions such as surface roughness or even systematic errors in the measurement (zero line offset) can produce similar signals from the metallic substrate.

\section{LIMITATIONS OF THE APPROACH}

It has been shown that, as conventional RAS is extended into the IR region, the anisotropic optical response of metallic nanostructures with a strong shape anisotropy, ranging from dense islands to nanowires, becomes significant. It was shown that established anisotropic EMA models, using the bulk response of the metal, cannot describe the response for the samples with the strongest infrared RAS signals. The anisotropic response can be modeled successfully, however, by a surface layer described by two Drude dielectric functions. In contrast to the determination of the bulk dielectric functions of metals, where the Drude response can be easily fitted using two parameters $\gamma$ and $\omega_{p}$, the description of the RAS signal needed four independent parameters $\left(\omega_{p}, \gamma_{x}, \gamma_{y}\right.$, and $\left.d\right)$ - too many for a unique determination of all the parameters. In the examples above, it was possible to fix some of the parameters, typically the thickness of the layer and the plasma frequency, using other data. The model could be extended to include percolation effects by combining an effective medium approach with the anisotropic formulation of the free electron gas. Such an extension, involving additional fitting parameters, could not be justified for the spectra discussed here because good agreement was obtained with the simpler model, except in regions where the spectra were dominated by interband transitions. For the same reason EMA models used for grid systems ${ }^{49}$ which might work well for aligned nanowires, were not explored in detail.

A significant limitation, however, becomes apparent when the IR RAS response is small, as in the case of anisotropic metal surfaces. The output from anisotropic EMA and surface layer Drude models may no longer be clearly distinguishable. It is anticipated that this problem can be alleviated by extending the spectral range further into the IR, where the free-electron-like response will be larger. It has already been shown by Goletti et al. that an extension to $0.3 \mathrm{eV}$ can be achieved with a standard polarizer/PEM setup. ${ }^{50}$ Conceptually different setups will have to be developed, however, to move further into the IR: one approach would be the use of an FTIR ellipsometer at near normal incidence.

The second limitation concerns the extrapolation of the high frequency conductance anisotropy, as measured by RAS, to obtain the dc sheet conductance, an important parameter for future applications of metallic nanowires. Measurements are only possible above $0.5 \mathrm{eV}$ with the current instrumentation, where the anisotropic free electron contribution may be too low, or the interband contribution too high, for reliable fitting. Additionally, the limited spectral range currently available may result in an error in the extrapolated dc value in cases where metallic nanostructures have significant percolation effects around the experimental cutoff of $0.5 \mathrm{eV}^{39}$

The last problem is the limitation of the Drude free electron model itself, which leads to a dielectric function involving a merely phenomenological scattering parameter. However, it is possible to extend the model by allowing for screening $\left(\varepsilon_{\infty}\right)$ and by using the effective mass of the electrons $m^{*}$ instead of the free electron mass, an approach used to describe the Drude absorption by free carriers in semiconductors. ${ }^{51}$ Anisotropic parameters are likely to be necessary, however, for modeling the RAS response. Indeed, for $\operatorname{Si}(111): \operatorname{In}-(4 \times 1)$, it is already known that there is an anisotropy in the effective mass of the electrons and hence the plasma frequency $\omega_{p}$ in the metallic band. ${ }^{52}$ This more sophisticated model could not be applied because the total number of parameters becomes too large to be useful (in the absence of additional data allowing some of these to be fixed), and the spectral range is already too small for definitive fits even with the simpler model. In the case of the tin islands, where the free electron contribution was shown to be dominant, fits allowing for an anisotropic $\omega_{p}$ produced a $\omega_{p, y} / \omega_{p, x}$ ratio that varied between 0.97 and 1.03 , 
showing that the anisotropy in the scattering rate is indeed more dominant $\left(\gamma_{y} / \gamma_{x}\right.$ between 1.1 and 1.5) as was suggested in the Introduction. The estimated dc anisotropy varies only by $10 \%$ and is not particularly sensitive to the details of the model, as the line shape can be reproduced in both cases.

\section{CONCLUSIONS}

It has been shown that, by extending the spectral range into the IR, it becomes feasible to use RAS as an optical probe of conductance anisotropies in metallic nanostructures. In the case of isolated or dense anisotropic metallic islands on semiconducting substrates, this anisotropic, free-electronlike contribution may even dominate the RAS spectra. If the underlying bulk is also metallic, the anisotropic response of near surface free electrons is less clear and is difficult to distinguish from anisotropic roughness effects modeled using anisotropic EMAs. For even smaller structures, such as atomic nanowires, the free electron response is small compared to anisotropic interband contributions. For these types of systems, extension of the spectral range further into the IR will be necessary to obtain reliable results.

So far only metallic overlayers, which have already been investigated by other techniques, have been studied. As RAS can also probe buried structures, future experiments may be able to reveal if the conductance anisotropy of nanowires is changed when they are covered by protective layers.

\section{ACKNOWLEDGMENTS}

The authors would like to acknowledge financial support for this work provided by the Irish Higher Education Authority, IRCSET Grant No. SC/2003/223/, and the SFB 290 of the Deutsche Forschungsgemeinschaft.
*Electronic address: fleisck@tcd.ie

${ }^{1}$ F. J. Himpsel, K. N. Altmann, R. Bennewitz, J. N. Crain, A. Kirakosian, J. L. Lin, and J. L. McChesney, J. Phys.: Condens. Matter 13, 11097 (2001), and references therein.

${ }^{2}$ H. W. Yeom, S. Takeda, E. Rotenberg, I. Matsuda, K. Horikoshi, J. Schaefer, C. M. Lee, S. D. Kevan, T. Ohta, T. Nagao, and S. Hasegawa, Phys. Rev. Lett. 82, 4898 (1999).

${ }^{3}$ P. Segovia, D. Purdie, M. Hengsberger, and Y. Baer, Nature (London) 402, 504 (1999).

${ }^{4}$ M. G. Zacher, E. Arrigoni, W. Hanke, and J. R. Schrieffer, Phys. Rev. B 57, 6370 (1998).

${ }^{5}$ D. E. Aspnes and A. A. Studna, Phys. Rev. Lett. 54, 1956 (1985).

${ }^{6}$ V. I. Berkovits, I. V. Makarenko, T. A. Minashvili, and V. I. Safarov, Solid State Commun. 56, 449 (1985).

${ }^{7}$ D. E. Aspnes, J. P. Harbison, A. A. Studna, and L. T. Florez, J. Vac. Sci. Technol. A 6, 1327 (1988).

${ }^{8}$ I. Kamiya, D. Aspnes, H. Tanaka, L. Florez, E. Colas, J. Harbison, and R. Bhat, Appl. Surf. Sci. 60-61, 534 (1992).

${ }^{9}$ M. A. Herman, W. Richter, and H. Sitter, Epitaxy, No. 62 in Springer Series in Materials Science (Sringer-Verlag, Berlin, 2004).

${ }^{10}$ O. Pulci, M. Palummo, M. Marsili, and R. Sole, Adv. Solid State Phys. 45, 161 (2005).

${ }^{11}$ W. G. Schmidt, K. Seino, P. H. Hahn, E. Bechstedt, W. Lu, S. Wang, and J. Bernholc, Thin Solid Films 455-56, 764 (2004), and references therein.

${ }^{12}$ J. R. Power, P. Weightman, and J. D. O’Mahony, Phys. Rev. B 56, 3587 (1997).

${ }^{13}$ F. Pedreschi, J. D. O'Mahony, P. Weightman, and J. R. Power, Appl. Phys. Lett. 73, 2152 (1998).

${ }^{14}$ K. Fleischer, S. Chandola, N. Esser, W. Richter, and J. F. McGilp, Phys. Rev. B 67, 235318 (2003).

${ }^{15}$ S. Wang, W. Lu, W. G. Schmidt, and J. Bernholc, Phys. Rev. B 68, 035329 (2003).

${ }^{16}$ P. Grosse, Freie Elektronen im Festkörper (Springer, New York 1979).

${ }^{17}$ P. Johnson and R. Christy, Phys. Rev. B 6, 4370 (1972).
${ }^{18}$ P. Monachesi, M. Palummo, R. DelSole, R. Ahuja, and O. Eriksson, Phys. Rev. B 64, 115421 (2001).

${ }^{19}$ J. D. McIntyre and D. E. Aspnes, Surf. Sci. 24, 417 (1971).

${ }^{20}$ O. Hunderi, J.-T. Zettler, and K. Haberland, Thin Solid Films 472, 261 (2005).

${ }^{21}$ S. Berthier, J. Phys. I 4, 303 (1994).

${ }^{22}$ J.-T. Zettler, J. Rumberg, K. Ploska, K. Stahrenberg, M. Pristovsek, W. Richter, M. Wassermeier, P. Schutzendube, J. Behrend, and L. Däweritz, Phys. Status Solidi A 152, 35 (1995).

${ }^{23}$ P. K. L. Drude, Ann. Phys. 1, 556 (1900).

${ }^{24}$ A. H. Wilson, Proc. R. Soc. London, Ser. A 133, 458 (1931).

${ }^{25}$ N. W. Ashcroft and N. D. Mermin, Solid State Physics (Thomson Learning, Inc., London, 1976).

${ }^{26}$ M. A. Ordal, L. L. Long, R. J. Bell, S. E. Bell, R. R. Bell, J. R. W. Alexander, and C. A. Ward, Appl. Opt. 22, 1099 (1983).

${ }^{27}$ J. Bremer, J.-K. Hansen, and O. Hunderi, Appl. Surf. Sci. 142, 286 (1999).

${ }^{28}$ V. Mazine, Y. Borensztein, L. Cagnon, and P. Allongue, Phys. Status Solidi A 175, 311 (1999).

${ }^{29}$ T. Herrmann, M. Gensch, M. J. G. Lee, A. I. Shkrebtii, N. Esser, W. Richter, and P. Hofmann, Phys. Rev. B 69, 165406 (2004).

${ }^{30}$ The sign in Eq. (5) depends on the sign in the ansatz for the electromagnetic plane waves. The convention of Ref. 19 is followed, with $\mathbf{E}(t)=\operatorname{Re}\left[\mathbf{E}(\omega) e^{\omega t}\right]$, leading to $\widetilde{\varepsilon}=\varepsilon^{\prime}-i \varepsilon^{\prime \prime}$.

${ }^{31}$ F. E. Hutchison and W. N. Hansen, Phys. Rev. B 20, 4076 (1979).

${ }^{32}$ G. Fahsold, A. Priebe, N. Magg, and A. Pucci, Thin Solid Films 428, 107 (2003).

${ }^{33}$ M. Stróżak, V. Hnatyuk, and M. Jałochowski, Vacuum 74, 241 (2004).

${ }^{34}$ R. A. MacRae and E. T. Arakawa, Phys. Rev. 162, 162 (1967).

${ }^{35}$ N. Esser, M. Frisch, A. Röseler, S. Schintke, B. O. Fimland, and C. Goletti, Phys. Rev. B 67, 125306 (2003).

${ }^{36}$ D. Stroud, Phys. Rev. B 19, 1783 (1979).

${ }^{37}$ J. Szczyrbowski and K. Schmalzbauer, J. Phys. F: Met. Phys. 16, 2079 (1986).

${ }^{38}$ B. Shinozaki, K. Omata, K. Yamada, T. Kawaguti, Y. Fujimori, and T. Aomine, Physica C 296, 49 (1998). 
${ }^{39}$ G. Fahsold, M. Sinther, A. Priebe, S. Diez, and A. Pucci, Phys. Rev. B 70, 115406 (2004).

${ }^{40}$ M. Gurnett, J. B. Gustafsson, K. O. Magnusson, S. M. Widstrand, and S. O. L. Johansson, Phys. Rev. B 66, 161101(R) (2002), and references therein.

${ }^{41}$ S. Chandola, J. Jacob, K. Fleischer, P. Vogt, W. Richter, and J. McGilp, J. Phys.: Condens. Matter 18, 6979 (2006), and references therein.

${ }^{42}$ X. López-Lozano, A. Stekolnikov, J. Furthmüller, and F. Bechstedt, Surf. Sci. 589, 77 (2005).

${ }^{43}$ T. Kanagawa, R. Hobara, I. Matsuda, T. Tanikawa, A. Natori, and S. Hasegawa, Phys. Rev. Lett. 91, 036805 (2003).

${ }^{44}$ Please note that $\sigma_{\|}$from Ref. 43 corresponds to $d \operatorname{Re}\left(\widetilde{\sigma}_{x}\right)$ and $\sigma_{\perp}$ to $d \operatorname{Re}\left(\widetilde{\sigma}_{y}\right)$ in our notation.
${ }^{45}$ K. Fleischer, S. Chandola, N. Esser, and J. McGilp, Phys. Status Solidi B 242, 2655 (2005).

${ }^{46}$ J.-K. Hansen, J. Bremer, and O. Hunderi, Phys. Status Solidi A 170, 271 (1998).

${ }^{47}$ D. Martin, S. Barrett, and P. Weightman, J. Phys.: Condens. Matter 13, 9847 (2001).

${ }^{48}$ J. Bremer and O. Hunderi, Phys. Status Solidi A 184, 89 (2001).

${ }^{49}$ T. Sergan, M. Lavrentovich, J. Kelly, E. Gardner, and D. Hansen, J. Opt. Soc. Am. A 19, 1872 (2002).

${ }^{50}$ C. Goletti, G. Bussetti, F. Arciprete, P. Chiaradia, and G. Chiarotti, Phys. Rev. B 66, 153307 (2002).

${ }^{51}$ P. Y. Yu and M. Cardona, Fundamentals of Semiconductors 2nd ed. (Sringer-Verlag, Berlin, 1999).

${ }^{52}$ I. G. Hill and A. B. McLean, Phys. Rev. Lett. 82, 2155 (1999). 